\title{
Molecular Characterization of Firework-Related Urban Aerosols using FT-ICR Mass Spectrometry
}

Qiaorong $\mathrm{Xie}^{1,8}$, Sihui $\mathrm{Su}^{2}$, Shuang $\mathrm{Chen}^{2}$, Yisheng $\mathrm{Xu}^{3}$, Dong $\mathrm{Cao}^{4}$, Jing Chen ${ }^{5}$, Lujie Ren ${ }^{2}$, Siyao Yue ${ }^{1,6,8}$, Wanyu Zhao ${ }^{1,8}$, Yele Sun ${ }^{1}$, Zifa Wang ${ }^{1}$, Haijie Tong 6 , Hang Su${ }^{6}$, Yafang Cheng ${ }^{6}$, Kimitaka Kawamura $^{7}$, Guibin Jiang ${ }^{4}$, Cong-Qiang Liu ${ }^{2}$, and Pingqing $\mathrm{Fu}^{2}$

${ }^{1}$ State Key Laboratory of Atmospheric Boundary Layer Physics and Atmospheric Chemistry, Institute of Atmospheric 10 Physics, Chinese Academy of Sciences, Beijing 100029, China

${ }^{2}$ Institute of Surface-Earth System Science, Tianjin University, Tianjin 300072, China

${ }^{3}$ State Key Laboratory of Environmental Criteria and Risk Assessment, Chinese Research Academy of Environmental Sciences, Beijing 100012, China

${ }^{4}$ State Key Laboratory of Environmental Chemistry and Ecotoxicology, Research Center for Eco-Environmental Science,

15 Chinese Academy of Sciences, Beijing 100085, China

${ }^{5}$ School of Environmental Science and Engineering, Tianjin University, Tianjin, 300072, China

${ }^{6}$ Max Planck Institute for Chemistry, Multiphase Chemistry Department, Hahn-Meitner-Weg 1, 55128 Mainz, Germany

${ }^{7}$ Chubu Institute for Advanced Studies, Chubu University, Kasugai 487-8501, Japan

${ }^{8}$ College of Earth and Planetary Sciences, University of Chinese Academy of Sciences, Beijing 100049, China

20 Correspondence to: Pingqing Fu (fupingqing@tju.edu.cn)

This supplementary information document contains 12 pages including 3 tables, 7 figures and references. 
Table S1. Number of compounds in each subgroup and arithmetic and weighted mean elemental ratio for each subgroup in LNY D and LNY N samples.

\begin{tabular}{|c|c|c|c|c|c|}
\hline & & All & $\mathrm{CHO}$ & $\mathrm{CHNO}$ & $\mathrm{CHOS}$ \\
\hline \multirow[t]{12}{*}{ LNY D } & Number frequency & 9511 & 3120 & 3604 & 1249 \\
\hline & Molecular weight $(\mathrm{Da})$ & $448 \pm 97$ & $456 \pm 120$ & $472 \pm 112$ & $402 \pm 82$ \\
\hline & $\mathrm{O} / \mathrm{C}$ & $0.35 \pm 0.14$ & $0.31 \pm 0.12$ & $0.33 \pm 0.11$ & $0.40 \pm 0.13$ \\
\hline & $\mathrm{O} / \mathrm{C}_{\mathrm{w}}$ & 0.36 & 0.31 & 0.33 & 0.39 \\
\hline & $\mathrm{H} / \mathrm{C}$ & $1.18 \pm 0.36$ & $1.14 \pm 0.37$ & $1.08 \pm 0.29$ & $1.37 \pm 0.43$ \\
\hline & $\mathrm{H} / \mathrm{C}_{\mathrm{w}}$ & 1.18 & 1.10 & 1.05 & 1.46 \\
\hline & $\mathrm{OM} / \mathrm{OC}$ & $1.65 \pm 0.22$ & $1.50 \pm 0.17$ & $1.60 \pm 0.16$ & $1.80 \pm 0.21$ \\
\hline & $\mathrm{OM} / \mathrm{OC}_{\mathrm{w}}$ & 1.66 & 1.50 & 1.60 & 1.79 \\
\hline & DBE & $11.2 \pm 4.98$ & $12.2 \pm 5.95$ & $13.2 \pm 4.97$ & $7.21 \pm 4.65$ \\
\hline & $\mathrm{DBE}_{\mathrm{w}}$ & 10.8 & 11.8 & 13.1 & 6.05 \\
\hline & $\mathrm{DBE} / \mathrm{C}$ & $0.47 \pm 0.17$ & $0.47 \pm 0.18$ & $0.53 \pm 0.14$ & $0.37 \pm 0.21$ \\
\hline & $\mathrm{DBE} / \mathrm{C}_{\mathrm{w}}$ & 0.48 & 0.50 & 0.55 & 0.33 \\
\hline \multirow[t]{12}{*}{ LNY N } & Number frequency & 8426 & 2618 & 2515 & 1626 \\
\hline & Molecular weight (Da) & $413 \pm 85$ & $420 \pm 100$ & $415 \pm 86$ & $402 \pm 78$ \\
\hline & $\mathrm{O} / \mathrm{C}$ & $0.34 \pm 0.13$ & $0.28 \pm 0.12$ & $0.34 \pm 0.11$ & $0.34 \pm 0.16$ \\
\hline & $\mathrm{O} / \mathrm{C}_{\mathrm{w}}$ & 0.35 & 0.28 & 0.34 & 0.31 \\
\hline & $\mathrm{H} / \mathrm{C}$ & $1.28 \pm 0.38$ & $1.24 \pm 0.40$ & $1.14 \pm 0.34$ & $1.42 \pm 0.42$ \\
\hline & $\mathrm{H} / \mathrm{C}_{\mathrm{w}}$ & 1.28 & 1.24 & 1.08 & 1.50 \\
\hline & $\mathrm{OM} / \mathrm{OC}$ & $1.66 \pm 0.20$ & $1.47 \pm 0.16$ & $1.61 \pm 0.17$ & $1.71 \pm 0.25$ \\
\hline & $\mathrm{OM} / \mathrm{OC}_{\mathrm{w}}$ & 1.67 & 1.48 & 1.61 & 1.68 \\
\hline & DBE & $9.19 \pm 4.8$ & $9.98 \pm 5.23$ & $11.0 \pm 4.50$ & $6.80 \pm 4.30$ \\
\hline & $\mathrm{DBE}_{\mathrm{w}}$ & 8.71 & 9.38 & 11.3 & 5.81 \\
\hline & $\mathrm{DBE} / \mathrm{C}$ & $0.43 \pm 0.20$ & $0.42 \pm 0.20$ & $0.51 \pm 0.17$ & $0.34 \pm 0.21$ \\
\hline & $\mathrm{DBE} / \mathrm{C}_{\mathrm{w}}$ & 0.43 & 0.43 & 0.54 & 0.31 \\
\hline
\end{tabular}


Table S2. Number of compounds in each subgroup and arithmetic and weighted mean elemental ratio for each subgroup in Normal D and Normal N samples.

\begin{tabular}{|c|c|c|c|c|c|}
\hline & & All & $\mathrm{CHO}$ & $\mathrm{CHNO}$ & $\mathrm{CHOS}$ \\
\hline \multirow[t]{12}{*}{ Normal D } & Number frequency & 5945 & 2168 & 2378 & 1399 \\
\hline & Molecular weight (Da) & $405 \pm 80$ & $400 \pm 90$ & $406 \pm 79$ & $405 \pm 83$ \\
\hline & $\mathrm{O} / \mathrm{C}$ & $0.36 \pm 0.15$ & $0.31 \pm 0.12$ & $0.34 \pm 0.10$ & $0.38 \pm 0.15$ \\
\hline & $\mathrm{O} / \mathrm{C}_{\mathrm{w}}$ & 0.36 & 0.31 & 0.34 & 0.36 \\
\hline & $\mathrm{H} / \mathrm{C}$ & $1.26 \pm 0.38$ & $1.14 \pm 0.38$ & $1.10 \pm 0.34$ & $1.44 \pm 0.40$ \\
\hline & $\mathrm{H} / \mathrm{C}_{\mathrm{w}}$ & 1.29 & 1.12 & 1.11 & 1.54 \\
\hline & $\mathrm{OM} / \mathrm{OC}$ & $1.69 \pm 0.22$ & $1.51 \pm 0.15$ & $1.62 \pm 0.16$ & $1.77 \pm 0.24$ \\
\hline & $\mathrm{OM} / \mathrm{OC}_{\mathrm{w}}$ & 1.69 & 1.51 & 1.62 & 1.76 \\
\hline & DBE & $9.01 \pm 4.25$ & $10.6 \pm 4.95$ & $11.0 \pm 4.26$ & $6.49 \pm 4.06$ \\
\hline & $\mathrm{DBE}_{\mathrm{w}}$ & 8.59 & 10.6 & 10.9 & 5.38 \\
\hline & $\mathrm{DBE} / \mathrm{C}$ & $0.44 \pm 0.19$ & $0.47 \pm 0.19$ & $0.53 \pm 0.17$ & $0.34 \pm 0.20$ \\
\hline & $\mathrm{DBE} / \mathrm{C}_{\mathrm{w}}$ & 0.42 & 0.49 & 0.52 & 0.29 \\
\hline \multirow[t]{12}{*}{ Normal N } & Number frequency & 5454 & 2071 & 2140 & 1243 \\
\hline & Molecular weight (Da) & $416 \pm 88$ & $408 \pm 99$ & $414 \pm 89$ & $395 \pm 81$ \\
\hline & $\mathrm{O} / \mathrm{C}$ & $0.37 \pm 0.14$ & $0.31 \pm 0.11$ & $0.34 \pm 0.11$ & $0.41 \pm 0.14$ \\
\hline & $\mathrm{O} / \mathrm{C}_{\mathrm{w}}$ & 0.38 & 0.31 & 0.34 & 0.41 \\
\hline & $\mathrm{H} / \mathrm{C}$ & $1.24 \pm 0.36$ & $1.19 \pm 0.37$ & $1.11 \pm 0.30$ & $1.36 \pm 0.42$ \\
\hline & $\mathrm{H} / \mathrm{C}_{\mathrm{w}}$ & 1.23 & 1.16 & 1.09 & 1.40 \\
\hline & $\mathrm{OM} / \mathrm{OC}$ & $1.70 \pm 0.19$ & $1.51 \pm 0.15$ & $1.61 \pm 0.17$ & $1.81 \pm 0.22$ \\
\hline & $\mathrm{OM} / \mathrm{OC}_{\mathrm{w}}$ & 1.72 & 1.51 & 1.61 & 1.82 \\
\hline & DBE & $9.71 \pm 4.47$ & $10.2 \pm 4.77$ & $11.6 \pm 4.20$ & $7.17 \pm 4.48$ \\
\hline & $\mathrm{DBE}_{\mathrm{w}}$ & 9.20 & 10.0 & 11.4 & 6.49 \\
\hline & $\mathrm{DBE} / \mathrm{C}$ & $0.46 \pm 0.18$ & $0.45 \pm 0.18$ & $0.52 \pm 0.15$ & $0.38 \pm 0.20$ \\
\hline & $\mathrm{DBE} / \mathrm{C}_{\mathrm{w}}$ & 0.46 & 0.47 & 0.53 & 0.36 \\
\hline
\end{tabular}


Table S3. Comparison of chemical characterization of water-soluble organic compounds in aerosol samples.

\begin{tabular}{|c|c|c|c|c|c|c|}
\hline Sampling site & Compounds & $\mathrm{O} / \mathrm{C}$ & $\mathrm{H} / \mathrm{C}$ & DBE & $\mathrm{DBE} / \mathrm{C}$ & Ref. \\
\hline Non-firework & All & $0.37 \pm 0.14$ & $1.24 \pm 0.37$ & $9.36 \pm 4.42$ & $0.45 \pm 0.18$ & This study \\
\hline Firework & All & $0.37 \pm 0.13$ & $1.23 \pm 0.37$ & $10.1 \pm 4.82$ & $0.45 \pm 0.18$ & This study \\
\hline Free tropospheric & All & $0.53 \pm 0.2$ & $1.48 \pm 0.3$ & $6.18 \pm 3.0$ & NA & (Mazzoleni et al., 2012) \\
\hline Free tropospheric & All & $0.46 \pm 0.13$ & $1.17 \pm 0.26$ & $10.7 \pm 4.0$ & $0.47 \pm 0.14$ & (Dzepina et al., 2015) \\
\hline Rural & All & $0.46 \pm 0.23$ & $1.34 \pm 0.39$ & $5.3 \pm 2.6$ & $0.45 \pm 0.21$ & (Lin et al., 2012) \\
\hline Rural & All & $0.28-0.32$ & $1.37-1.46$ & $6.30-7.45$ & $0.33-0.38$ & (Wozniak et al., 2008) \\
\hline Marin boundary layer & All & $0.36-0.42$ & $1.49-1.56$ & $5.88-6.76$ & $0.28-0.32$ & (Wozniak et al., 2014) \\
\hline Remote & All & $0.39-0.42$ & $1.30-1.34$ & $7.71-8.38$ & $0.41-0.42$ & (An et al., 2019) \\
\hline Free tropospheric & $\mathrm{CHO}$ & $0.47 \pm 0.2$ & $0.47 \pm 0.2$ & $0.47 \pm 0.2$ & NA & (Mazzoleni et al., 2012) \\
\hline Free tropospheric & $\mathrm{CHO}$ & $0.47 \pm 0.14$ & $1.19 \pm 0.27$ & $10.8 \pm 4.3$ & $0.46 \pm 0.14$ & (Dzepina et al., 2015) \\
\hline Rural & $\mathrm{CHO}$ & $0.40 \pm 0.21$ & $1.29 \pm 0.35$ & $5.6 \pm 2.4$ & $0.44 \pm 0.18$ & (Lin et al., 2012) \\
\hline Urban (hazy) & $\mathrm{CHO}$ & $0.41 \pm 0.19$ & $1.19 \pm 0.38$ & $8.0 \pm 3.9$ & $0.47 \pm 0.19$ & (Jiang et al., 2016) \\
\hline Free tropospheric & $\mathrm{CHNO}$ & $0.57 \pm 0.2$ & $0.57 \pm 0.2$ & $6.72 \pm 2$ & NA & (Mazzoleni et al., 2012) \\
\hline Free tropospheric & $\mathrm{CHNO}$ & $0.45 \pm 0.10$ & $1.14 \pm 0.22$ & $10.3 \pm 2.9$ & $0.51 \pm 0.12$ & (Dzepina et al., 2015) \\
\hline Rural & CHNO & $0.41 \pm 0.19$ & $1.15 \pm 0.31$ & $6.4 \pm 2.1$ & $0.59 \pm 0.16$ & (Lin et al., 2012) \\
\hline Urban (hazy) & CHNO & $0.45 \pm 0.22$ & $1.13 \pm 0.38$ & $8.8 \pm 4.0$ & $0.55 \pm 0.19$ & (Jiang et al., 2016) \\
\hline Free tropospheric & $\mathrm{CHOS}$ & $0.56 \pm 0.2$ & $1.64 \pm 0.3$ & $1.64 \pm 0.3$ & NA & (Mazzoleni et al., 2012) \\
\hline Free tropospheric & CHOS & $0.50 \pm 0.11$ & $1.75 \pm 0.31$ & $3.5 \pm 2.6$ & $0.2 \pm 0.14$ & (Dzepina et al., 2015) \\
\hline Rural & $\mathrm{CHOS}$ & $0.55 \pm 0.17$ & $1.67 \pm 0.31$ & $3.0 \pm 1.9$ & $0.25 \pm 0.16$ & (Lin et al., 2012) \\
\hline Urban (hazy) & $\mathrm{CHOS}$ & $0.65 \pm 0.28$ & $1.64 \pm 0.37$ & $3.4 \pm 2.4$ & $0.26 \pm 0.18$ & (Jiang et al., 2016) \\
\hline
\end{tabular}


Daytime
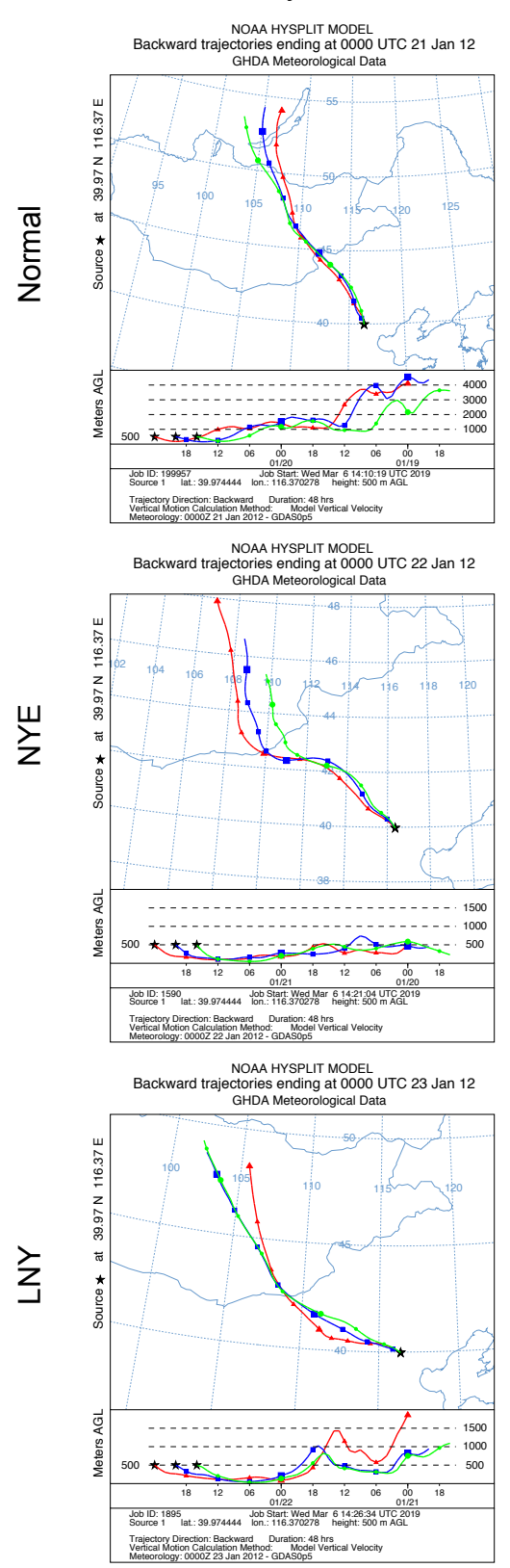

Nighttime

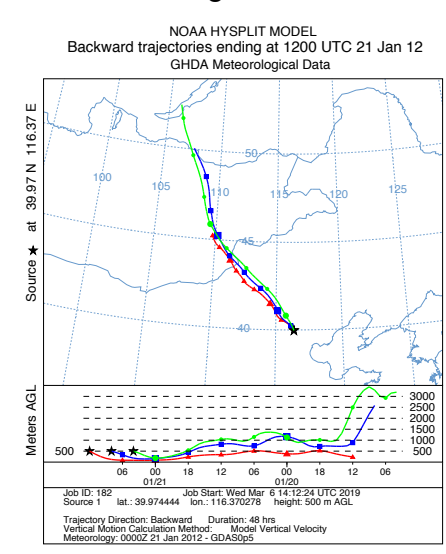

NOAA HYSPLIT MODEL
Backward trajectories ending at 1200 UTC 22 Jan 12

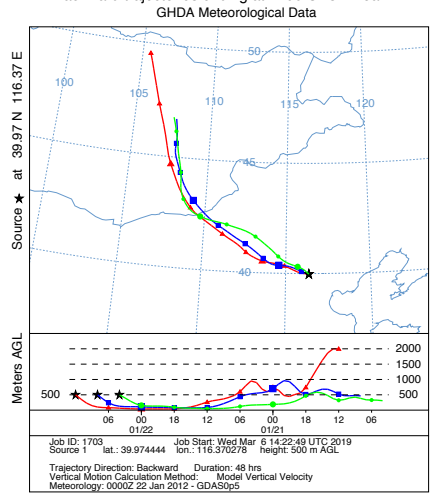

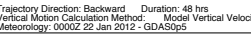
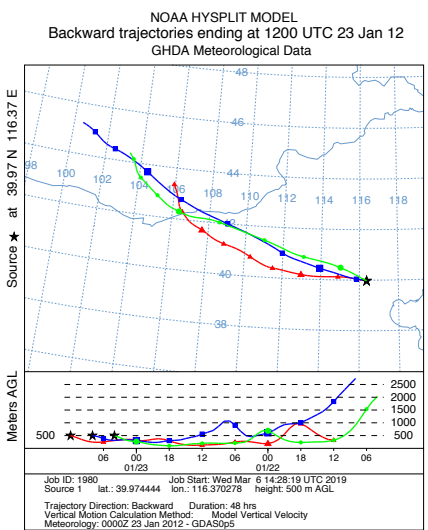

Figure S1: The clustering air mass two-day backward trajectories. 


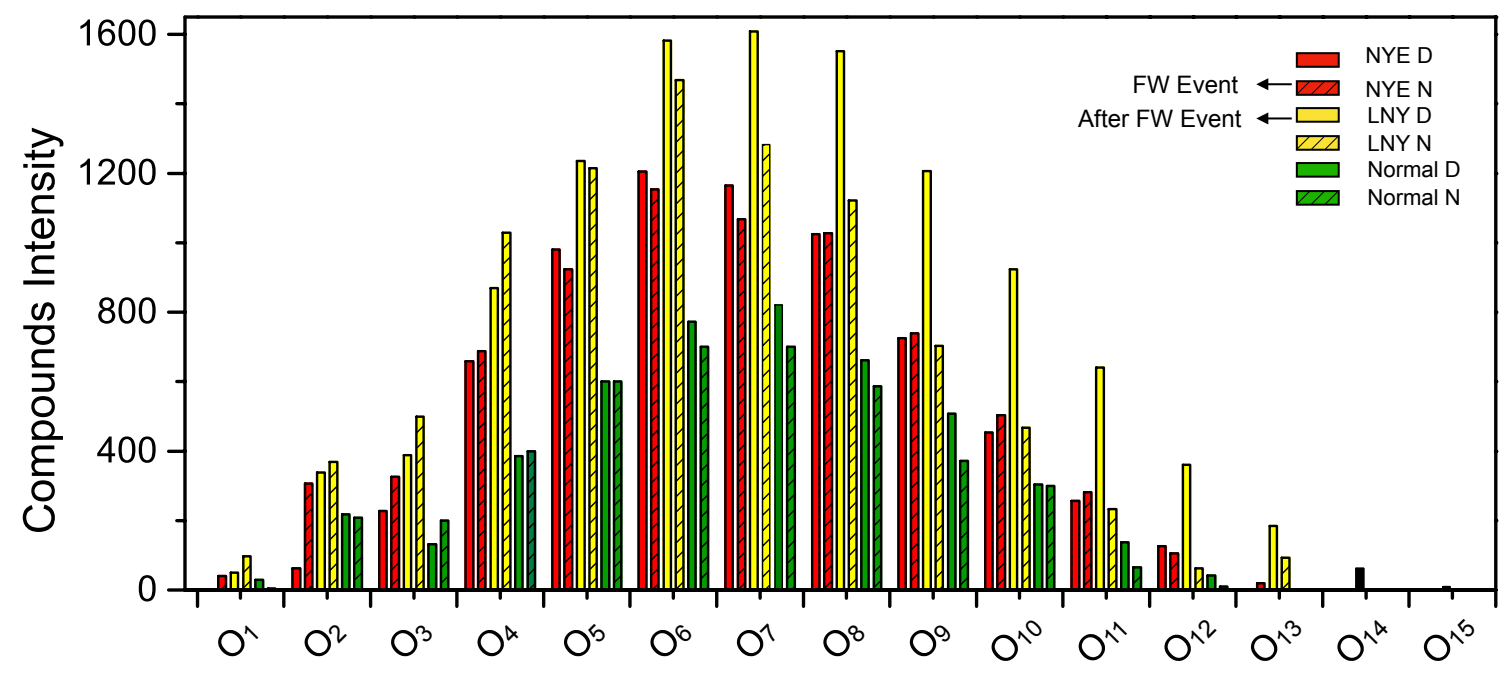

Figure S2: Intensity of CHO species of subgroups according to the number of $\mathrm{O}$ atoms in their molecules. 

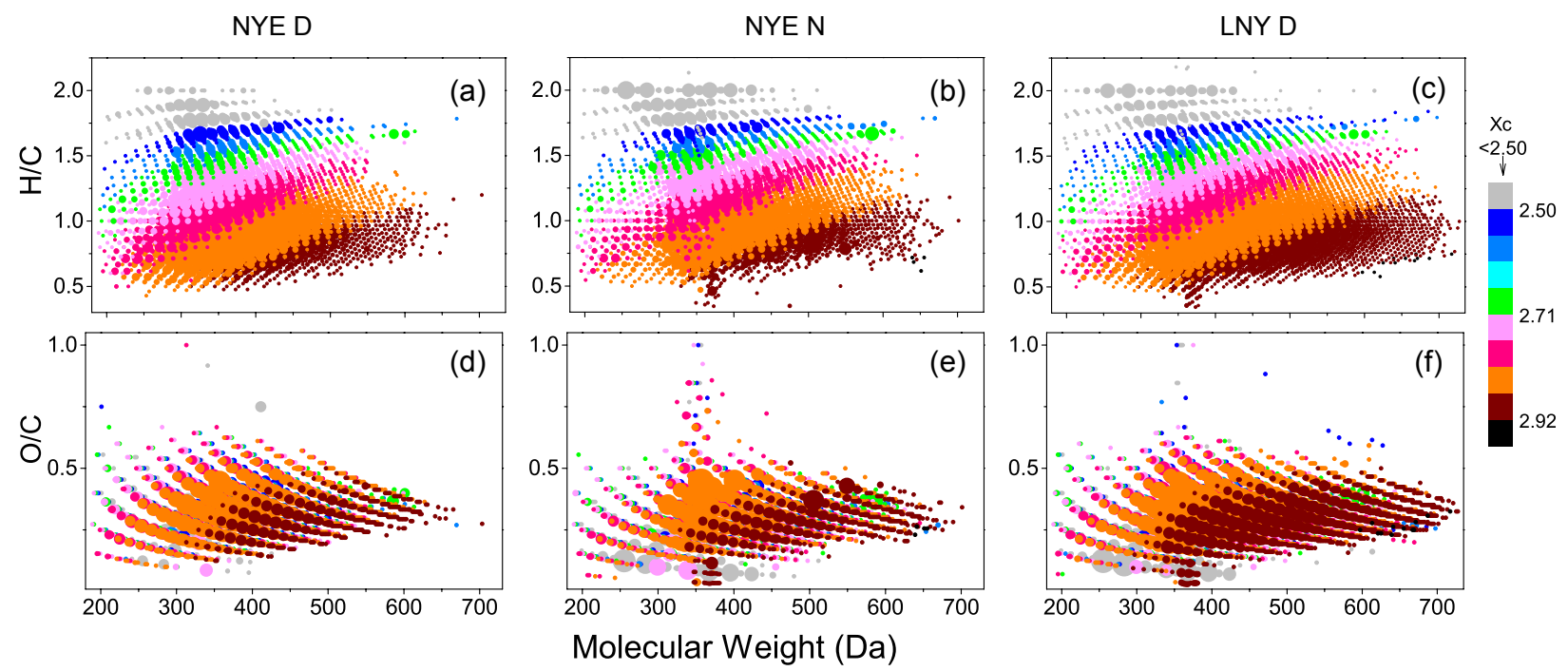

Figure S3: The $\mathrm{H} / \mathrm{C}(\mathbf{a}, \mathbf{b}, \mathbf{c})$ and $\mathrm{O} / \mathrm{C}(\mathbf{d}, \mathbf{e}, \mathbf{f})$ ratios of $\mathrm{CHO}$ formulae are shown as a function of their neutral mass from NYE D (a, d), NYE N (b, e) and LNY D $(\mathbf{c}, \mathbf{f})$ samples with their $\mathrm{X}_{\mathrm{c}}$ values color-coded. Grey data points indicate non-aromatic compounds $\left(\mathrm{X}_{\mathrm{c}}<2.5\right)$, blue to green data $\left(2.5<\mathrm{X}_{\mathrm{c}}<2.71\right)$ are mono-aromatic compounds and pink to black data $\left(\mathrm{X}_{\mathrm{c}}>2.71\right)$ includes PAHs. The size of the symbols reflects the relative peak intensities of molecular formulae on a logarithmic scale. 

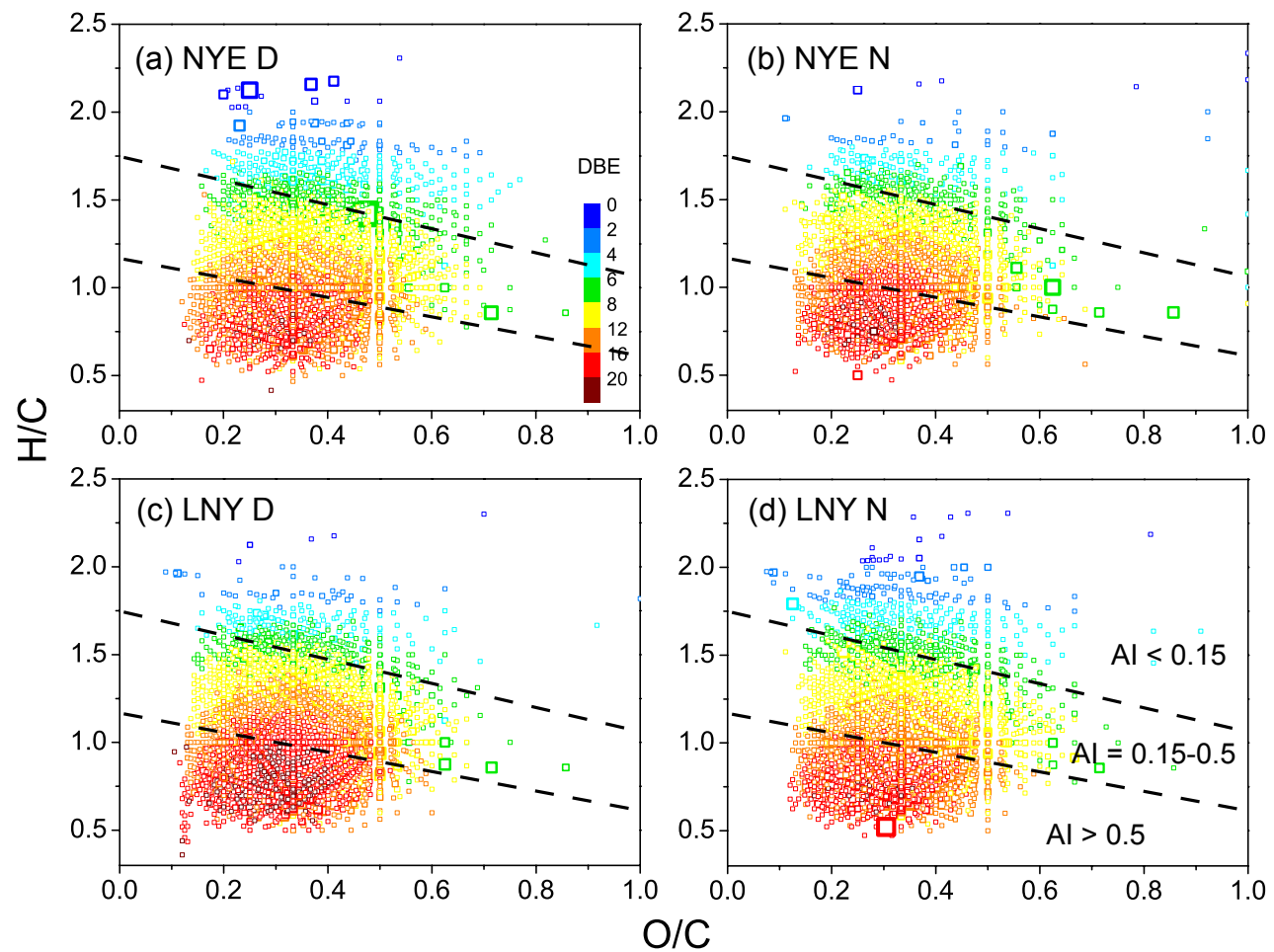

Figure S4: Van Krevelen diagrams (the $\mathrm{H} / \mathrm{C}$ via $\mathrm{O} / \mathrm{C}$ ratios) for the $\mathrm{CHNO}$ compounds with various aromatic index (AI) values ranges. The dashes lines separate the different AI regions. The size of the symbols reflects the relative peak intensities of compounds on a logarithmic scale. 


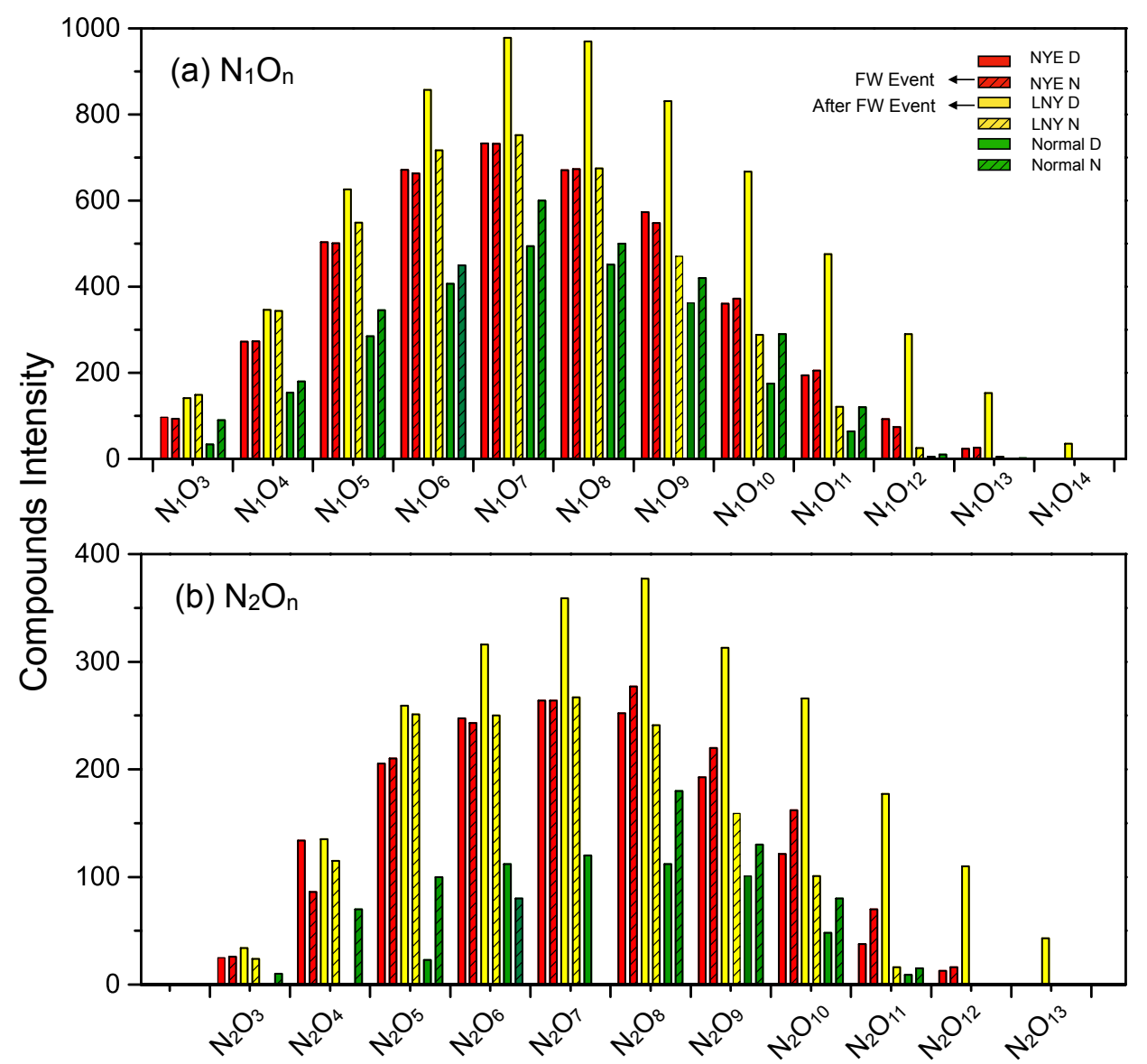

Figure S5: Intensity of CHNO species of subgroups according to the number of $\mathrm{N}$ and $\mathrm{O}$ atoms in their molecules. 


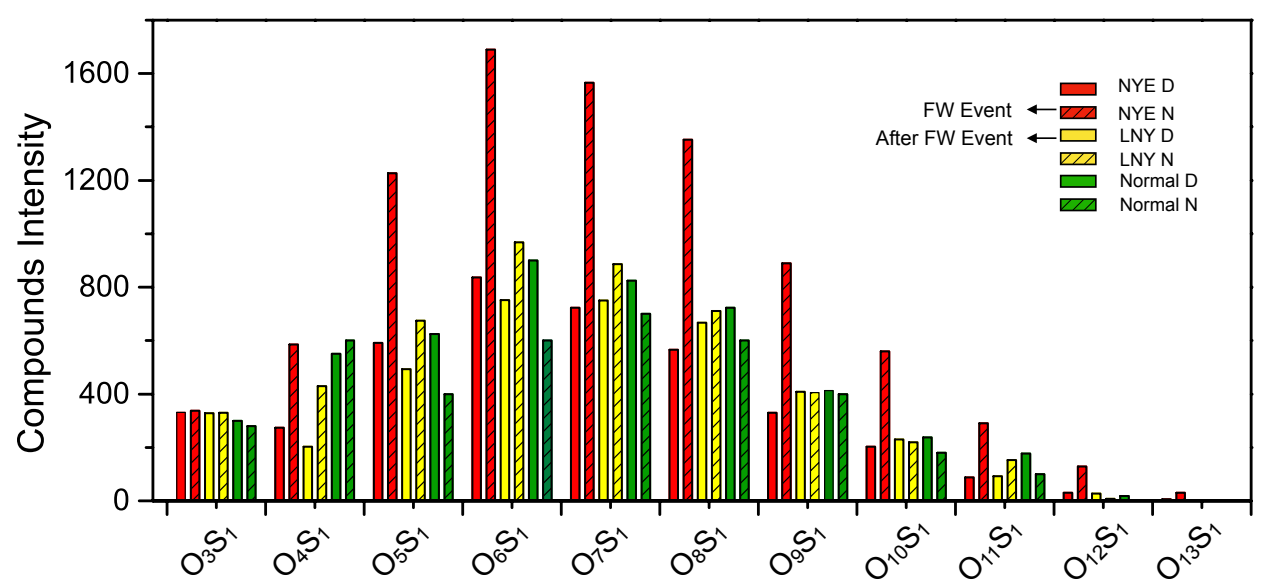

Figure S6: Intensity of CHOS species of subgroups according to the number of $\mathrm{O}$ and $\mathrm{S}$ atoms in their molecules. 

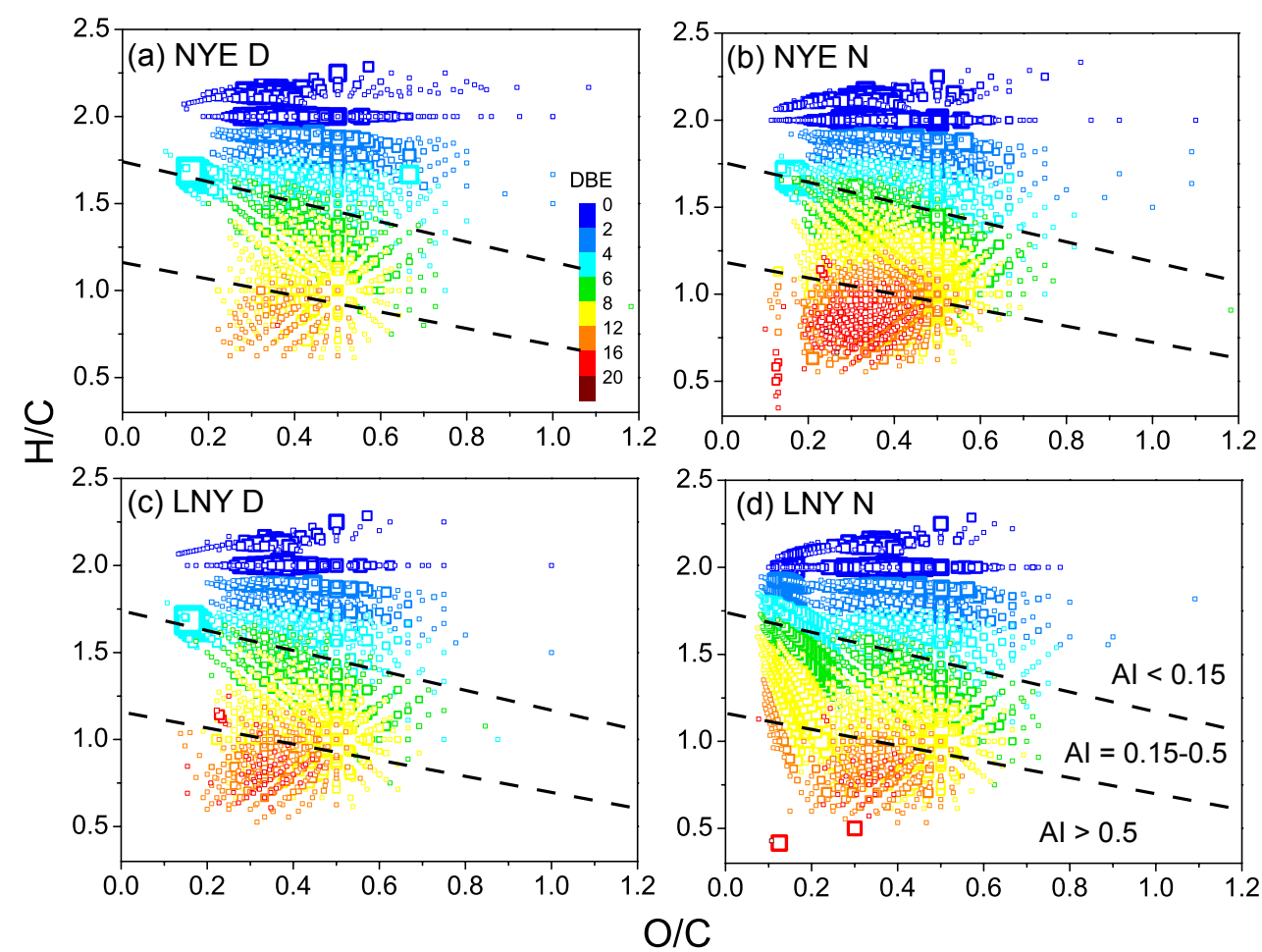

Figure S7: Van Krevelen diagrams (the $\mathrm{H} / \mathrm{C}$ via $\mathrm{O} / \mathrm{C}$ ratios) for the $\mathrm{CHOS}$ compounds with various aromatic index (AI) values ranges. The dashes lines separate the different AI regions. The size of the symbols reflects the relative peak intensities of compounds on a 5 logarithmic scale. 


\section{References}

An, Y. Q., Xu, J. Z., Feng, L., Zhang, X. H., Liu, Y. M., Kang, S. C., Jiang, B., and Liao, Y. H.: Molecular characterization of organic aerosol in the Himalayas: insight from ultra-high-resolution mass spectrometry, Atmos. Chem. Phys., 19, 1115-1128, 10.5194/acp-191115-2019, 2019.

5 Dzepina, K., Mazzoleni, C., Fialho, P., China, S., Zhang, B., Owen, R. C., Helmig, D., Hueber, J., Kumar, S., Perlinger, J. A., Kramer, L. J., Dziobak, M. P., Ampadu, M. T., Olsen, S., Wuebbles, D. J., and Mazzoleni, L. R.: Molecular characterization of free tropospheric aerosol collected at the Pico Mountain Observatory: a case study with a long-range transported biomass burning plume, Atmos. Chem. Phys., 15, 5047-5068, 10.5194/acp-15-5047-2015, 2015.

Jiang, B., Kuang, B. Y., Liang, Y., Zhang, J., Huang, X. H., Xu, C., Yu, J. Z., and Shi, Q.: Molecular composition of urban organic 10 aerosols on clear and hazy days in Beijing: a comparative study using FT-ICR MS, Environ. Chem., 13, 888-901, 2016.

Lin, P., Rincon, A. G., Kalberer, M., and Yu, J. Z.: Elemental composition of HULIS in the Pearl River Delta Region, China: Results inferred from positive and negative electrospray high resolution mass spectrometric data, Environ. Sci. Technol., 46, 7454-7462, 2012.

Mazzoleni, L. R., Saranjampour, P., Dalbec, M. M., Samburova, V., Hallar, A. G., Zielinska, B., Lowenthal, D. H., and Kohl, S.: Identification of water-soluble organic carbon in non-urban aerosols using ultrahigh-resolution FT-ICR mass spectrometry: Organic anions,

15 Environ. Chem., 9, 285-297, 2012.

Wozniak, A. S., Bauer, J. E., Sleighter, R. L., Dickhut, R. M., and Hatcher, P. G.: Technical Note: Molecular characterization of aerosolderived water soluble organic carbon using ultrahigh resolution electrospray ionization Fourier transform ion cyclotron resonance mass spectrometry, Atmos. Chem. Phys., 8, 5099-5111, 10.5194/acp-8-5099-2008, 2008.

Wozniak, A. S., Willoughby, A. S., Gurganus, S. C., and Hatcher, P. G.: Distinguishing molecular characteristics of aerosol water soluble organic matter from the 2011 trans-North Atlantic US GEOTRACES cruise, Atmos. Chem. Phys., 14, 8419-8434, 2014. 